\title{
Addressing early childhood development and developmental difficulties in Turkey: a training program for developmental pediatrics units
}

\author{
Emine B. Bingoler Pekcici, M.D. ${ }^{a}$, Ezgi Özalp Akin, M.D. ${ }^{a}$, Iclal Ayranci Sucakli, M.D. ${ }^{b}$, \\ Gizem Kara Elitok, M.D. ${ }^{c}$, Fatma N. Onat, M.D. ${ }^{d}$, Meltem Kivilcim, M.D. ${ }^{e}$, \\ Zeynep Ustunyurt, M.D. ${ }^{f}$, Revan Mustafayev, M.D. ${ }^{a}$ and Ilgi Ozturk Ertem, M.D. ${ }^{a}$
}

a. Ankara University School of Medicine, Department of Pediatrics, DevelopmentalBehavioral Pediatrics Division, Ankara, Turkey.

b. Ankara Child Health and Diseases, HematologyOncology Training and Research Hospital, University of Health Sciences, Ankara, Turkey.

c. Sisli Hamidiye Etfal Training and Research Hospital, University of Health Sciences, Istanbul, Turkey.

d. Sami Ulus Maternity and Children's Training and Research Hospital, Ankara, Turkey.

e. Behcet Uz Children's Training and Research Hospital, Izmir, Turkey.

f. Zekai Tahir Burak Women's Health Training and Research Hospital, University of Health Sciences, Ankara, Turkey.

E-mail address:

Emine B. Bingoler

Pekcici M.D.:

baharbingoler@yahoo.

com

Funding:

None.

Conflict of interest:

None.

Received: 4-26-2019

Accepted: 11-21-2019

\section{ABSTRACT}

Introduction. Addressing early childhood development (ECD) and developmental difficulties (DDs) in low and middle-income countries (LMICs) requires applicable and sustainable programs. Seven years after its implementation in 2010, we evaluated the Developmental Pediatrics Unit (DPU) Training Program in pediatric referral centers in Turkey. Population and methods. We applied crosssectional design and quantitative/qualitative methods to assess services, training, research and advocacy of the DPUs.

Results. Five of six established DPUs sustained clinical services, training, research, and advocacy. A total of 23,710 children (9085 new cases in 2017) had been referred mainly for perinatal risks and chronic illness, all centers contributed with similar proportions of children. Staff motivation and endurance, hospital administrator support, and client satisfaction facilitated the program; whereas invisibility within the performance based healthcare system was a barrier.

Conclusions. In Turkey and possibly other LMICs, the DPU Training Program is applicable and sustainable if health system barriers are addressed.

Key words: child development, disabled children, low income population.

http:/ / dx.doi.org/10.5546/ aap.2020.eng.e384

To cite: Bingoler Pekcici EB, Özalp Akin E, Ayranci Sucakli I, Kara ElitokG, et al. Addressing early childhood development and developmental difficulties in Turkey: a training program for developmental pediatrics units. Arch Argent Pediatr 2020;118(4):e384-e391.

\section{INTRODUCTION}

The recently published World Health Organization Nurturing Care Framework defines "developmental difficulties" (DDs) as any condition that puts a child at risk of suboptimal development, or that causes a child to have a developmental deviance, delay, disorder or disability. The term encompasses all children who have limitations in functioning and developing to their full potential. ${ }^{1}$ The United Nations Sustainable Development Goals 2030 include access to quality early childhood development (ECD) for all children, emphasizing the importance of addressing ECD and DDs within healthcare systems. ${ }^{2}$ Most children, and $95 \%$ of those with DDs live in low and middle-income countries (LMICs). ${ }^{3}$ Child and infant mortality, the past focus of healthcare systems in LMICs is decreasing ${ }^{4}$ and strategies for addressing ECD and DDs are increasingly needed. ${ }^{2}$ Two decades ago, the seminal strategy document titled "Neurological, Psychiatric, and Developmental Disorders: Meeting the Challenge in the Developing World" highlighted the importance of strengthening referral centers to address DDs. ${ }^{5}$ While some information on how primary healthcare systems can address ECD exists, ${ }^{6}$ there is paucity of research on how DDs can be addressed in referral centers in LMICs. ${ }^{6}$

Research from several high-income countries (HICs), such as Australia, ${ }^{7}$ Israel, ${ }^{8}$ and United States ${ }^{9}$ suggest that pediatricians require training to address ECD and DDs. In such countries, this training is based on 
developmental-behavioral pediatrics (DBP), child psychiatry rotations, or both. ${ }^{10} \mathrm{~A}$ major barrier for pediatricians in LMICs has been the lack of opportunities for such training. ${ }^{11,12}$ Variations in developmental risk factors, ${ }^{2}$ the knowledge of caregivers, ${ }^{13}$ and clinicians ${ }^{14}$ may require training that specifically pertains to LMICs. Furthermore, multidisciplinary teams able to work together to provide comprehensive assessments, interventions in HICs may often not be available in referral centers in LMICs. ${ }^{12}$

To address the need for training programs, between 2005-2010, we developed the training package titled "Addressing Developmental Difficulties and Early Childhood Development $(\mathrm{ADD}+\mathrm{ECD})$ " which aims to improve the knowledge, skills and attitudes of health providers from primary care and referral centers in monitoring and supporting ECD; and the prevention, early identification, early intervention and management of DDs. ${ }^{15} \mathrm{We}$ have trained clinicians from over 30 countries in various $\mathrm{ADD}+\mathrm{ECD}$ components, in particular the International Guide for Monitoring Child Development (GMCD), a universal tool for the prevention, early identification of DDs and early intervention. ${ }^{14,16-18}$ We had previously reported on one of the ADD+ECD components, the threeday training program for primary healthcare providers which included counseling caregivers on child development; developmental monitoring for early identification, and management of DDs during routine health care encounters. Our qualitative research on this program in Turkey had revealed that consultations to referral centers by trained primary health providers were ineffective $\cdot{ }^{14}$ Referral centers lacked pediatricians who could provide comprehensive assessments and management of children with DDs and lacked specific space where such children could be served. ${ }^{14}$ We now report a second component of ADD+ECD, the Developmental Pediatrics Unit (DPU) Program. Different from the training for primary care, the DPU Program is designed to meet the needs of pediatric referral centers and aims to train a multidisciplinary team comprising pediatricians and clinicians from allied disciplines enabling them in establishing a DPU within the referral center. The purpose of this study was to evaluate seven years after the establishment of the DPUs, the clinical services, training, research and advocacy provided as a result of the DPU Program, as well as facilitators and barriers to DPU services.

\section{POPULATION AND METHODS The DPU Program}

The objectives of the three month DPU program were to provide trainees' updated knowledge, skills and attitudes related to addressing ECD and DDs and their ability to form and sustain a DPU in the referral center. These objectives included enhancing skills in: a) providing clinical services to promote ECD, recognize developmental risk factors, provide comprehensive family-centered developmental assessments, use standardized assessment tools, manage DDs with an individualized early intervention plan, and coordinate services; b) training pediatric residents, family physicians and other health care personell; c) research; and d) advocacy in ECD and DDs. Nurturing in the trainees persistent enthusiasm and devotion to learn and serve children and caregivers with humility was a main goal of the program.

One of us (IE) prepared the DPU training curriculum by studying existing curricula of programs on ECD and DDs and incorporated long-term experience so as to meet the needs in Turkey and potentially other LMICs. Based on bioecological theory of child development, ${ }^{19}$ relationship, strengths-based, and familycentered approaches, ${ }^{20}$ the World Health Organization (WHO) International Classification of Functioning, Disability and Health(ICF) framework, ${ }^{21}$ and transdisciplinary principles ${ }^{22}$ 180-hours of theoretical, and 240-hours of practicum sessions were applied. A complete list of theoretical seminars is provided in Table 1. References included a manual that was developed specifically for the training, the reference handbook "Developmental Pediatrics" in Turkish, articles, books, journals, PowerPoint slide-sets and educational videotapes.

The objectives of the practicum sessions were to enable clinicians to perform transdisciplinary developmental assessments using family-centered methods which enabled a diagnosis when possible, and a comprehensive early intervention plan based on the ICF framework (body structures, functions, activities and participation in life) to meet the child and family's needs. Diagnostic classification systems used included the WHO International Classification of Functioning, Disability and Health (ICF), Diagnostic and Statistical Manual of Mental Disorders $4^{\text {th }}$ Edition (DSM-IV), and the Zero to Three Diagnostic Classification of Mental Health and Developmental Disorders of Infancy and Early Childhood (DC:0-3). The tools used included the 
GMCD, Expanded GMCD which encompasses all ICF domains, Vineland Adaptive Behavior Scales Second Edition, the Bayley Scales of Infant and Toddler Development Second Edition, Home Observation Measurement of the Environment, Modified Checklist for Autism in Toddlers as well as measures of family mental health and functioning. Training included making efficient and effective referrals for specific testing (e.g. auditory, language, vision testing) or treatment (e.g. for cerebral palsy, autism, caregiver mental health problems) when needed. The practicum involved observing children and families in real life settings such as homes, hospitals, pre-schools, early intervention centers. Trainees observed mentors with backgrounds in developmentalbehavioral pediatrics, child psychiatry and psychology before conducting assessments and interventions. Direct observations by mentors or videotaping was used to provide reflective supervision. Under supervision, trainees assessed and followed a minimum of 50 children with a variety of DDs.

The training included an 8-hour course on research methodology. Advocacy projects pertained to improving practices within the health system to promote ECD and DDs, petitioning, attending parliament and ministries for legislation change, and working with not-forprofit organizations to fulfill the needs of children with special needs.

The DPU Program was instituted in 2010 in Turkey as a model of multilateral cooperation among academia, pediatric referral centers, the Ministry of Health (MoH), and United Nations Children's Emergency Fund (UNICEF). The entire training was conducted at Ankara University (AU) School of Medicine Department of Pediatrics, Developmental Pediatrics Division. One professor full-time, and two-hours each of five other academicians was devoted by AU. The administration of the referral centers signed a room for the DPUs, and the $\mathrm{MoH}$ provided staff salaries, UNICEF funded travel and accommodation for trainees living outside of Ankara and developmental tools for the DPUs. Out of 59 similar hospitals in the country, the $\mathrm{MoH}$ selected those with the greater number of inpatient beds and pediatric residents and the hospital administration chose the DPU Program trainees. Before the implementation of the program, trainees did not work as a team, and lacked previous training in ECD and DDs.

Table 1. Theoretical seminar topics of the Developmental Pediatrics Units Program

\section{Introduction to early childhood development}

Theories of child development (bioecological, attachment, motherhood constellation); stages of child development and parenthood (pregnancy, labor, infancy, early childhood, school age, adolescence and young adulthood); United Nations Convention on the Rights of Children, Convention on the Rights of Persons with Disabilities.

\section{Developmental risk factors and resilience}

Psychosocial risk factors (poverty, stress, parental depression, abuse and neglect, substance abuse, crisis situations and disasters); biological risk factors (malnutrition, anemia, iron deficiency, perinatal risk factors including prematurity, genetic, neurological, endocrine/metabolic disorders, other chronic illnesses and their effects on development); addressing risk factors and monitoring and supporting development during infancy, early childhood, school-age, adolescence, early adulthood.

\section{Principles of family-centered comprehensive developmental assessments}

Developmental history taking; developmental observation techniques; selection of instruments for different ages and contexts; assessment of the child's functioning, activities and participation, family and community context, services and systems; assessment process as an intervention, transitioning seamlessly to early intervention.

\section{Diagnostic Classification Systems}

WHO International Classification of Functioning Disability and Health; Diagnostic and Statistical Manual of Mental Disorders; International Classification of Diseases; Zero to Three Diagnostic Classification of Mental Health and Developmental Disorders of Infancy and Early Childhood.

Assessment, diagnoses, treatment, rehabilitation, case-management and follow-up of specific developmental disorders Language delay, autism spectrum disorder, cerebral palsy, central and peripheric nervous system disorders, cognitive, learning, speech, visual, hearing and multiple impairments; behavioral feeding, sleep disorders, enuresis, encopresis.

\section{Programs and systems to support development}

The role of the healthcare system, home and family-based programs, home visiting, community-based programs, rehabilitation center-based programs, transdisciplinary approaches, effective advocacy and program development in ECD. 
Pediatricians and clinicians from allied disciplines (psychologists, special educators) were eligible but due to staff inadequacy, only pediatricians and child development specialists were enrolled. The selection criteria included being interested in ECD and DDs; volunteering for the three months training; being regarded by the administration as a leading clinician working well with children and families.

\section{Study design and measures}

In this cross-sectional study, we applied both qualitative and quantitative methods. To evaluate the outcomes of the DPU program we developed a survey with both structured and open ended questions on the characteristics of the referral centers and services provided by the DPUs. The survey was finalized as an online SurveyMonkey tool and completed by the DPU pediatricians in January 2018. To obtain additional qualitative information, two of us (BBP and IE) carried out faceto-face individual interviews with staff of the DPUs, and administrators of hospitals where the DPUs were located. The study was approved by the Ethics committees of AU and all of the DPU institutions.

\section{Data analysis}

We used descriptive statistics to analyze the number of clinicians trained and the services they provided. The z-test for independent groups was conducted to determine whether proportions of reasons for referrals were statistically significant across the DPUs. An adjusted p value of $<0.05$ was considered statistically significant. Thematic qualitative analysis was performed on the openended questions and face-to-face interviews to investigate and describe the experiences of the DPU staff and administrators, their perceptions related to the training, the research and advocacy that was provided as well as facilitators and barriers. Two authors coded all responses to the open-ended questions and the transcripts from the interviews independently to group related concepts and identify key themes. Theme saturation was determined, and representative quotes were written. Discrepancies between coders were resolved through consensus.

\section{RESULTS \\ Characteristics of the pediatric referral centers and DPUs}

Trainees of the DPU Program were from six pediatric referral centers, all were trained at AU and all six DPUs were established in 2010. When the study was conducted in January 2018, five DPUs were still functioning. The DPUs were located in four metropoles with populations ranging 4-20 million, one each in Istanbul and Izmir, northern and western Turkey, respectively; and three in central and capital Ankara. These referral centers were children's hospitals with a median of 271 pediatric beds (IQR: 142-392), and 60 neonatal intensive care

TABLE 2. Reasons for referral to the Developmental Pediatrics Units

\begin{tabular}{|c|c|c|c|c|c|c|c|c|c|c|}
\hline \multirow[t]{2}{*}{ Reasons for referral $(\mathrm{N}=23,710)$} & \multicolumn{2}{|c|}{ DPU-1 } & \multicolumn{2}{|c|}{ DPU-2 } & \multicolumn{2}{|c|}{ DPU-3 } & \multicolumn{2}{|c|}{ DPU-4 } & \multicolumn{2}{|c|}{ DPU-5 } \\
\hline & $\mathbf{n}$ & $(\%)$ & $\mathbf{n}$ & $(\%)$ & $\mathbf{n}$ & $(\%)$ & $\mathbf{n}$ & $(\%)$ & $\mathbf{n}$ & $(\%)$ \\
\hline Perinatal problems and premature infant follow-up & 2696 & 44.8 & 1130 & 35.3 & 3094 & 46.4 & 436 & 33.4 & 5214 & 80.0 \\
\hline $\begin{array}{l}\text { Chronic developmental conditions (congenital, } \\
\text { genetic, metabolic and neurological disorders, } \\
\text { autism spectrum disorder, cerebral palsy, }\end{array}$ & & & & & & & & & & \\
\hline children from foster care) & 1534 & 25.5 & 871 & 27.2 & 1614 & 24.2 & 253 & 19.4 & 886 & 13.6 \\
\hline Speech and language delay & 1089 & 18.1 & 714 & 22.3 & 733 & 11.0 & 227 & 17.4 & 267 & 4.1 \\
\hline Behavioral problems & 385 & 6.4 & 195 & 6.1 & 640 & 9.6 & 109 & 8.3 & 104 & 1.6 \\
\hline Hearing and vision impairments & 18 & 0.3 & 0 & 0 & 13 & 0.2 & 9 & 0.7 & 46 & 0.7 \\
\hline Other developmental disorders & 90 & 1.5 & 0 & 0 & 287 & 4.3 & 0 & 0 & 0 & 0 \\
\hline $\begin{array}{l}\text { Children referred for developmental delay, } \\
\text { but identified as developing typically }\end{array}$ & 205 & 3.4 & 292 & 9.1 & 287 & 4.3 & 272 & 20.8 & 0 & 0 \\
\hline Total & 6017 & 100 & 3202 & 100 & 6668 & 100 & 1306 & 100 & 6517 & 100 \\
\hline
\end{tabular}

DPU-1: Turkish Ministry of Health (TmoH) Ankara Child Health and Diseases, Hematology-Oncology Training and Research Hospital; DPU-2: TmoH Dr. Behcet Uz Children's Training and Research Hospital, Izmir; DPU-3: TmoH Dr. Sami Ulus Maternity and Children's Training and Research Hospital, Ankara; DPU-4: TmoH Sisli Hamidiye Etfal Training and Research Hospital, Istanbul; DPU-5: TmoH Dr. Zekai Tahir Burak Women's Health Training and Research Hospital, Ankara. 
beds (IQR: 24-105); and served a median of 430 outpatient children per day (IQR: 173-790). The space allocated by the hospitals for the DPUs included one to three rooms, equipped with instruments for developmental assessments, and furniture to facilitate family-centered care. A oneway mirrored observation booth was present in three DPUs; one also had video-recording equipment. In total, six pediatricians and six child development specialists had been trained with the DPU Program. Of these, four pediatricians, and two child development specialists still were employed at the DPUs. By 2016, graduates of the newly established three-year subspecialty training in DBP were appointed by the $\mathrm{MoH}$ to three of the DPUs.

\section{Clinical services, training and research provided by the DPUs}

A total of 23,710 children and their families had received outpatient-inpatient services at the DPUs during 2011-2017, with 9,085 new cases in 2017. The DPUs had assessed a median of 9 children and families per day (IQR: 6-11) and 195 per month (IQR: 91-204). Most referrals to the DPUs were initiated by pediatricians who were working in the outpatient or inpatient clinics of the hospitals. Table 2 shows the reasons for referral. Perinatal problems were the most common reason and included children who were referred because they were born prematurely, with asphyxia or other problems and were at risk for developmental delay or who already had developmental delay due to perinatal causes. As can be seen in Table 2, the ranking of the proportions of reasons for referral were similar across all DPUs. When we analyzed with the $z$-test for differences in proportions the seven categories of reasons for referral of five DPUs, of the resulting 70 pairs, the proportions of children referred for each category of developmental difficulty was statistically significantly different for only 13 pairs $(19 \%)$.

All DPUs provided family-centered comprehensive assessments within one-month of a child's referral, depending on the urgency. The durations of the first assessment and follow-up visits were 40-60 and 20-30 minutes, respectively. The DPUs reported using the GMCD, Expanded GMCD, Vineland Adaptive Behavior Scales, and the Bayley Scales of Infant and Toddler Development most commonly from the tools provided in the training.
Training provided by the DPUs to residents and other clinicians were seminars and courses on ECD and DDs, training on the GMCD, and on rights and legislations related to DDs. A onemonth DPU rotation for residents had been instituted in only one institution, despite all five institutions providing four-year residency training. The DPUs had conducted 14 research projects over seven years. The DPUs advocated for a revision of disability legislations which did not differentiate children from adults, and for DP as a subspecialty. These efforts were far reaching and resulted in 2011 in the acceptance of Developmental Pediatrics as a subspecialty in Turkey, the establishment of the Turkish "Developmental Pediatrics Association". In 2015, the DPUs held the $1^{\text {st }}$ International Developmental Pediatrics Congress in Istanbul, and the International Developmental Pediatrics Association (IDPA) was formed. In 2019, DPU efforts resulted in the passing of the new legislation titled "Special Needs Legislation for Children" enabling assessment for child disability benefits using a non-stigmatizing, family-centered, ICF and needs-based system.

\section{Qualitative study results on facilitators and barriers to DPU services}

The qualitative study results indicated that the main facilitators for the program were the self-confidence, motivation and persistence of the DPU clinicians. The clinicians stated that their motivation was because of the adequacy of the DPU training, and the satisfaction of children and families they served. Clinicians and administrators stated that children with DDs and their families were referred to pediatric neurology or child psychiatry before but were not receiving the comprehensive assessments, and case-management that became possible in the DPUs. Another facilitator was support of the administrators of the hospitals who provided space and permitted allocation of time. Barriers included problems in reimbursement and sustainability of the staffing of the DPUs. Some of the trained clinicians had to be moved to other clinics by the administration due to the needs of the hospital. Administrators reported that the long duration of patient visits resulted in fewer number of children seen at the DPUs compared to other clinics, and was "a money-loser" in the performance-based healthcare system. The repeated theme was that DPU clinicians felt the obligation to prove the need for DPU services 
in pediatric settings that were still based on the medical model and focused on child survival.

\section{DISCUSSION}

This study reports the long-term results of the DPU Training Program related to addressing ECD and DDs in Turkey. Our findings indicate that the training program accomplished its goal to create sustainable DPUs in large pediatric referral centers. Seven years after the training, all but one of the DPUs that had been formed were still functioning and continue to provide services to large numbers of children with a variety of developmental risks and difficulties.

Studies of training programs for pediatricians in ECD and DD related concepts are mainly conducted in HICs where developmental services are a component of high-standard pediatric care. ${ }^{9,23}$ Research is emerging on examining curricula and models of training specific to pediatrics in $\mathrm{LMICs}^{24}$ and there is growing interest in training pediatricians in ECD and DDs. ${ }^{11}$ Two studies from Nepal demonstrate the gaps in knowledge and skills of pediatricians, and the need for training. ${ }^{25,26}$ Recent consensus statements from India ${ }^{27}$ address the need for training pediatricians in DDs. As mortality falls in LMICs ${ }^{4}$ and the pediatric community focuses on ECD and DDs, our study provides timely information on how training programs can be implemented.

The implications of our study pertain to the feasibility and sustainability of the DPU Program in Turkey but possibly other LMICs with similar health systems. Our findings indicate that the three-month DPU training was feasible; sustainability was demonstrated as after seven years, all but one of the DPUs were still functioning and the number of children receiving services increased over the years. Referrals included young children with DDs and perinatal problems were the leading reason for referral. This referral profile is different from reported reasons of referrals for developmental services in HICs, which are autism, attention deficit-hyperactivity disorder and learning disorders. ${ }^{10}$ This difference may be due to the ECD focus of the DPU Program but also because perinatal problems are recognized as the leading cause of developmental morbidity in LMICs. Four DPUs were typical and similar large training hospitals and the differences in the percentage of children referred to their DPUs were mostly similar whereas DPU-5 was a large maternity- newborn referral hospital which explains for the larger proportion of referrals for children with perinatal problems. These findings indicate that it may be possible for other centers to predict and plan trainings and services that can meet the needs of their referred population.

Our findings on sustainability align with studies that highlight the importance of trainee and personnel selection. ${ }^{28}$ The selected clinicians had volunteered for the training but had not been fully informed about receiving lower performance wages, nor asked for long-term commitment. Half of these clinicians had moved to other appointments. We recommend that candidates for such training programs should be chosen volunteering clinicians who are fully informed and dedicated for long-term commitment to the DPU.

The qualitative study findings on the main facilitators for sustainable services reemphasize the recommendations of the seminal North Carolina Assuring Better Child Health and Development Project which found that "identifying a physician champion to lead project activity" was a key element for success. ${ }^{29}$ This project also emphasized "aligning goals with collaborating partners to help ensure active participation from partners" the lack of which was a main barrier to DPU services. The collaborating partner $(\mathrm{MoH})$ policies were not aligned with the goal of investing time and resources for children with DDs. Health systems that recognize, value, and pay for services for ECD and DDs are crucial for initiatives like the DPU Program. Such programs would be strengthened if supported at scale by international organizations, in particular the WHO, UNICEF and the International Pediatric Association. The view by administrations that the DPU services are a "money loser" requires attention. Advocacy efforts to health ministries should focus on explaining that services of DPUs are in place to better meet the needs of each child and family and should be viewed as an ethical obligation and investment for the future of the country, not a short term financial venture.

The major strength of this study is that it provides the long-term results of a training program with qualitative and quantitative methods. The main limitation is that we were unable to apply an independent evaluation. Quantitative data such as number of patients seen would not have changed but it is unclear whether different facilitators and barriers could have been elicited through independent qualitative 
evaluations. Lack of data on referral reasons and how children with DDs were receiving care in the referral centers before the DPU Program was instituted is also a limitation of the study.

\section{CONCLUSIONS}

In this era when addressing ECD is incorporated within the United Nations Sustainable Development Goals, there is a great need for the advancement of addressing ECD and DDs in LMICs. The DPU Program applied and evaluated in Turkey, may be adapted to the needs and applied in other countries that may be facing similar challenges with regards to training in developmental pediatrics.

\section{REFERENCES}

1. World Health Organization, United Nations Children's Fund, World Bank Group. Nurturing care for early childhood development: a framework for helping children survive and thrive to transform health and human potential. Geneva: World Health Organization; 2018.

2. Black MM, Walker SP, Fernald LCH, Andersen CT, et al. Early childhood development coming of age: science through the life course. Lancet. 2017; 389(10064):77-90.

3. Global Research on Developmental Disabilities Collaborators. Developmental disabilities among children younger than 5 years in 195 countries and territories, 19902016: a systematic analysis for the Global Burden of Disease Study 2016. Lancet Glob Health. 2018; 6(10):e1100-21.

4. GBD 2015 Child Mortality Collaborators. Global, regional, national, and selected subnational levels of stillbirths, neonatal, infant, and under-5 mortality, 1980-2015: a systematic analysis for the Global Burden of Disease Study 2015. Lancet. 2016; 388(10053):1725-74.

5. Institute of Medicine (US), Committee on Nervous System Disorders in Developing Countries. Neurological, Psychiatric, and Developmental Disorders: Meeting the Challenge in the Developing World. Washington DC: National Academy Press; 2001.

6. Vaivada T, Gaffey MF, Bhutta ZA. Promoting Early Child Development with Interventions in Health and Nutrition: A Systematic Review. Pediatrics. 2017; 140(2):e20164308.

7. McDowell MJ, Lesslie JM. Long-term outcomes for children with neurodevelopmental disorders: Are they core business for paediatricians? J Paediatr Child Health. 2018; 54(5):469-73.

8. Porter B, Gadassi H, GrossmanZ, KeremE, etal.Community paediatrics in Israel: the 'Goshen' model for change. Arch Dis Child. 2017; 102(9):795-7.

9. Stein RE, Storfer-Isser A, Kerker BD, Garner A, et al. Does length of Developmental Behavioral Pediatrics training matter? Acad Pediatr. 2017; 17(1):61-7.

10. Haggerty RJ, Friedman SB. History of DevelopmentalBehavioral Pediatrics. J Dev Behav Pediatr. 2003; 24(Suppl 1):S1-18.

11. Kraus de Camargo OA. Children are the futuretheir development matters report. First International Developmental Pediatrics Congress. BJMHH. 2016; 4(1): 29-32.

12. World Health Organization. Developmental difficulties in early childhood: prevention, early identification, assessment and intervention in low and middle-income countries: A review. Geneva: WHO; 2012.
13. Ertem IO, Atay G, Dogan DG, Bayhan A, et al. Mothers' knowledge of young child development in a developing country. Child Care Health Dev. 2007; 33(6):728-37.

14. Ertem IO, Pekcici EB, Gok CG, Ozbas S, et al. Addressing early childhood development in primary health care: experience from a middle-income country. J Dev Behav Pediatr. 2009; 30(4):319-26.

15. Mustafayev R, Seyid-Mammadova T, Gulumser O, Balta H, et al. ADD-ECD: A Cascading Package To Address Developmental Difficulties And Early Childhood Development. Book of Abstracts. 2nd International Developmental Pediatrics Association Congress. 7-10 Dec 2017. Mumbai, India. 2017:13.

16. Ertem IO, Dogan DG, Gok CG, Kizilates SU, et al. A guide for monitoring child development in low- and middleincome countries. Pediatrics. 2008; 121(3):e581-9.

17. Ertem IO, Krishnamurthy V, Mulaudzi MC, Sguassero Y, et al. Similarities and differences in child development from birth to age 3 years by sex and across four countries: a cross-sectional, observational study. Lancet Glob Health. 2018; 6(3):e279-91.

18. Ertem IO, Krishnamurthy V, Mulaudzi MC, Sguassero Y, et al. Validation of the International Guide for Monitoring Child Development demonstrates good sensitivity and specificity in four diverse countries. Acta Paediatr. 2019; 108(6):1074-86.

19. BronfenbrennerU,CeciSJ. Nature-nurture reconceptualized in developmental perspective: A bioecological model. Psychol Rev. 1994; 101(4):568-86.

20. Committee on Hospital Care and Institute for Patient- And Family-Centered Care. Patient- and family-centered care and the pediatrician's role. Pediatrics.2012; 129(2):394-404.

21. World Health Organization. International Classification of Functioning, Disability and Health (ICF). Geneva: WHO; 2001.

22. Bell A, Corfield M, Davies J, Richardson N. Collaborative transdisciplinary intervention in early years- putting theory into practice. Child Care Health Dev. 2010; 36(1):142-8.

23. Kuo AA, Inkelas M, Maidenberg M, Lotstein DS, et al. Pediatricians' roles in the provision of developmental services: an international study. J Dev Behav Pediatr. 2009; 30(4):331-9.

24. Ganapathi L, Martins Y, SchumannD, Russ C. Overcoming challenges to develop pediatric postgraduate training programs in low- and middle-income countries. Educ Health (Abingdon). 2014; 27(3):277-82.

25. Shrestha M, Shrestha R. Symptom recognition to diagnosis of autism in Nepal. J Autism Dev Disord. 2014; 44(6):1483-5.

26. Rimal HS, Pokharel A, Saha V. Study on developmentalbehavioural pediatrics training experiences of pediatricians and pediatric trainees working in Nepal. Kathmandu Univ Med J (KUMJ). 2013; 11(43):191-5.

27. National Consultation Meeting for developing Indian Academy of Pediatrics (IAP), Guidelines on NeurodevelopmentalDisorders under the aegis of IAP Childhood Disability Group and the Committee on Child Development and Neurodevelopmental Disorders, C Nair MK, Prasad C, et al. Consensus Statement of the Indian Academy of Pediatrics on Evaluation and Management of Learning Disability. Indian Pediatr. 2017; 54(7):574-80.

28. Bassett ML, Ramsey WP, Chan CC. Improving medical personnel selection and appointment processes. Int J Health Care Qual Assur. 2012; 25(5):442-52.

29. Earls MF, Hay SS. Setting the stage for success: implementation of developmental and behavioral screening and surveillance in primary care practice-the North Carolina Assuring Better Child Health and Development (ABCD) Project. Pediatrics. 2006; 118(1):e183-8. 